Revue de droit comparé du travail et de la sécurité sociale

2| 2018

Harcèlement moral au travail en jurisprudence

comparée

\title{
Le droit du travail au-delà des frontières nationales : Les débats doctrinaux de l'année 2016
}

Steven L. Willborn, Eri Kasagi et Sebastián de Soto Rioja

\section{OpenEdition}

Journals

Édition électronique

URL : https://journals.openedition.org/rdctss/1965

DOI : $10.4000 /$ rdctss. 1965

ISSN : 2262-9815

Éditeur

Centre de droit comparé du travail et de la sécurité sociale

Édition imprimée

Date de publication : 1 juin 2018

Pagination : 126-149

ISSN : 2117-4350

Référence électronique

Steven L. Willborn, Eri Kasagi et Sebastián de Soto Rioja, «Le droit du travail au-delà des frontières nationales : Les débats doctrinaux de l'année $2016 »$, Revue de droit comparé du travail et de la sécurité sociale [En ligne], 2 I 2018, mis en ligne le 01 novembre 2021, consulté le 12 novembre 2021. URL http://journals.openedition.org/rdctss/1965 ; DOI : https://doi.org/10.4000/rdctss.1965

\section{(c) (i) $\ominus$}

Revue de droit comparé du travail et de la sécurité sociale est mise à disposition selon les termes de la Licence Creative Commons Attribution - Pas d'Utilisation Commerciale - Pas de Modification 4.0 International. 


\section{STEVEN L. WILLBORN}

Spencer Professeur de droit, Faculté de droit de I'Université du Nebraska, Etats-Unis

\section{ERI KASAGI}

Chargée de recherche CNRS, Centre de droit comparé du travail et de la sécurité sociale (COMPTRASEC), UMR 5114 CNRS - Université de Bordeaux, France

SEBASTIÁN dE SOTO RIOJA

Professeur de droit du travail et de la sécurité sociale,

Université Pablo de Olavide (Séville), Espagne

L'Association internationale des revues du droit du travail (IALLJ ou l'Association) est un consortium de 29 revues du monde entier ${ }^{1}$. Chaque année, depuis 2012, un groupe de rédacteurs collabore pour fournir une revue des principaux thèmes traités dans les articles publiés dans les journaux membres du consortium. En l'espèce, nous avons passé en revue plus de 700 articles qui ont été publiés dans les journaux de l'Association durant l'année 2016.

Comme on pouvait s'y attendre, les articles couvrent pratiquement toute la gamme des sujets liés au travail et à l'emploi. Travaillant avec une équipe exceptionnelle de rédacteurs ${ }^{2}$, nous avons été en mesure de trier les articles par thématiques et de sélectionner trois thèmes majeurs qui ont attiré une grande attention : l'efficacité de l'application des lois du travail (I), les défis posés par la nature changeante de l'emploi (II) et enfin, les questions liées aux salaires, à la pauvreté et aux revenus (III) ${ }^{3}$.

1 La liste complète des revues membres de l'IALLJ peut être consultée ici: www.labourlawjournals.

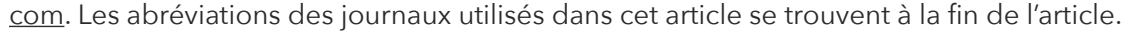

2 Les auteurs ont été assistés par une équipe de collègues qui ont répertorié, revu, catégorisé et (si nécessaire) traduit tous les articles. Les membres de cette équipe étaient Gian Guido Balandi, Antonio Pedro Baylos Grau, Marialaura Birgillito, Silvia Borelli, Matteo Borzaga, Isabelle Daugareilh, Manuel Garcia-Muñoz Alhambra, Eva Maria Hohnerlein, Barbara Kresal, Sandrine Laviolette, Miguel Rodríguez-Piñero Royo, Anna Rita Tinti, et Jesús Cruz Villalón.

3 Cette revue est le produit du travail commun des auteurs. Mais Sebastián de Soto Rioja a eu était principalement la responsabilité de la section A, Steve Willborn de la section B, et Eri Kasagi de la section $\mathrm{C}$. 


\section{LitTÉRATURE DE Droit SOCIAL COMPARÉ}

\section{I - L'EFFICACITÉ DE L'APPLICATION DES LOIS DU TRAVAIL}

Depuis les origines du droit du travail - ou de la "législation ouvrière », "Legislazione Operaia ", "Factory Acts " ou "Leyes de Fábrica " - il a été nécessaire de s'assurer que les prescriptions légales soient respectées, afin de garantir la réalisation effective de ses principaux objectifs : maintenir la paix et promouvoir la justice sociale. La mise en œuvre doit être particulièrement efficace pour les droits essentiels considérés comme étant à la base de l'ordre public (ius minimum), dans la mesure où ils affectent les moyens de subsistance des travailleurs, leur santé et même leur vie, en bref, leur dignité comme êtres humains. Le droit du travail n'est donc pas seulement une branche spécifique du droit; il adopte des positions critiques vis-à-vis du droit civil ou du droit commun et exige l'intervention de l'Etat.

Ces caractéristiques déterminantes expliquent pourquoi le droit du travail occupe une place prépondérante parmi les grands objectifs politiques: sa portée dépasse la simple économie et le rôle de la population active comme facteur de production, comme en témoigne l'existence depuis des siècles de ministères spécialisés ou d'agences spécialisées au sein des gouvernements du monde entier ainsi que dans différentes organisations internationales.

En effet, un corps de l'administration a été spécifiquement mis en place pour garantir l'application effective des normes du travail, telles que les inspections du travail et de la sécurité sociale. Ces corps de fonctionnaires sont présents dans plus de cent Etats ${ }^{4}$. Leur caractéristique principale est leur pouvoir de contrôle, d'alerte et/ou de sanction des infractions détectées, ce qui les place en première ligne de l'effectivité du droit du travail.

Cependant le contrôle et l'intervention administrative, ne sont pas la seule réponse de la loi à la "question sociale ». Comme l'a montré l'un des premiers experts internationaux du droit du travail, Anton Menger ${ }^{5}$, pour que la législation sociale soit vraiment efficace, elle a également besoin de l'action judiciaire, car les droits subjectifs ne deviennent réellement impératifs (ius cogens) que lorsque leur but et leurs conséquences sont judiciairement protégés. Ainsi, les conflits du travail sont généralement traités de manière singulière dans tous les systèmes judiciaires; dans la plupart des cas sont prévues des procédures spéciales distinctes, voire une juridiction civile distincte, commune ou administrative (lorsqu'il y en a une). Ces processus visent à garantir : la facilité d'accès, souvent au moyen de la gratuité pour les travailleurs et la reconnaissance de droits syndicaux de représentation, une plus grande simplicité des procédures, avec principalement des procédures orales, parfois avec

4 http://www.iali-aiit.org, site web de l'International Association of Labour Inspection.

5 Auteur de The Right to the Whole Produce of Labour (version anglaise, Londres-New York, 1899) est surtout connu pour son travail critique Das bürgerliche Recht und die besitzlosen Volksklassen: Eine Kritik des Entwurfs eines bürgerlichen Gesetzbuches für das deutsche Reich, Tübingen, ed. H. Laupp, 1890. 
dispense d'avocats, et une résolution rapide des litiges, étant donné que ce qui est habituellement en jeu dans le litige c'est la subsistance même des travailleurs et de leurs familles ${ }^{6}$.

II n'est pas surprenant que ces questions soient récurrentes dans la littérature scientifique liée à ce corpus juridique, a fortiori, si l'on inclut l'éventail des procédures légales qui répondent aux exigences «de l'efficacité» ou «de l'efficience » des normes du travail. On en veut pour exemple l'inclusion d'un plus grand nombre de sujets financièrement responsables en cas d'implication de plusieurs entreprises, ou encore l'extension du principe de solidarité des peines et non celle de la simple subsidiarité en cas d'insolvabilité du débiteur principal, ou la création de fonds publics garantissant les revenus auxquels ont droit les travailleurs, les assurés sociaux et leurs ayants droits.

En raison des effets de la mondialisation et du fait que les travailleurs sont considérés comme des citoyens à part entière, ces procédures juridiques doivent également intégrer l'internationalisation de la législation du travail et l'élever à la catégorie des droits fondamentaux de l'homme. Cela pose toutefois des problèmes supplémentaires, de coexistence avec des réalités comme le principe de territorialité et, par conséquent, de cohabitation de multiples lois du travail applicables sur un même territoire, parfois au sein d'une même Nation. Cela suppose aussi une harmonisation des qualifications juridiques et des différentes constructions jurisprudentielles.

Par ailleurs, les conditions de travail réelles sont généralement définies en dehors du droit formel, à travers des sources supplémentaires telles que les conventions collectives voire les contrats individuels de travail. C'est pourquoi, l'efficacité des normes conventionnelles et contractuelles doit également être garantie, et leur existence même exige un système de libertés, à savoir la liberté d'association et d'action collective, dont fait partie le droit de grève. Pour les travailleurs, les conditions de vie dépendent la plupart du temps de ces accords.

Sur l'ensemble des articles publiés par l'Association en 2016, plus d'une centaine, (soit un sur sept) se réfèrent directement et explicitement à des problèmes d'application des normes du travail. Les normes du travail ne sont pas analysées en l'espèce selon leur capacité à organiser le comportement ou à transformer la société dans le sens ontologique de ce qui « devrait être fait », en suivant les idéaux de justice sociale et redistributive?.

6 F. Valdés Dal-Ré, « Un examen comparatista de las jurisdicciones sociales: la convergencia en los principios, »DRL, 2017-6, p. 473.

7 Les articles sur cet aspect ont été nombreux en 2016 vue que c'était une période de réforme juridique majeure et de grandes transformations économiques. Voir B. Caruso, «Impresa, lavoro, diritto nella stagione del Jobs Act», DLRI, 2016, n 150, p. 255, D. Gesualdi Facteau, « De l'usage du droit du travail: une contribution théorique aux études portant sur l'effectivité du droit», RDCTSS, 2016-1, p. 16, E.M. Hohnerlein, « Soziale Rechte für Drittstaatsangehörige: Zugang zu Sozialleistungen aus unions- und menschenrechtlicher Perspektive», ZIAS, 2016-1, vol. 30, p. 47. U. Romagnoli, « Momenti di storia della cultura giuridica del lavoro», LD, 2016-1, p. 3, dans le même numéro, A. Perulli « L'idea di diritto del lavoro, oggi, » p. 17. A. Supiot, «What International Social Justice in the twenty-first century?», $B C L R, 2016, n^{\circ} 92$, p. 1 (disponible en français et en espagnol sur http://www.college-de-france.fr/site/alain-supiot/articles-en-ligne.htm), B. Veneziani, " Del contenuto essenziale dei diritti dei lavoratori: spunti per una ricerca», DLM, 2016-2, p. 229. 
Ce serait en effet difficile à évaluer. Il s'agit plutôt d'observer ici un ensemble de mécanismes juridiques et d'institutions destinés à garantir que les effets juridiques obligatoires prévus par les dispositifs légaux et réglementaires ont de réelles répercussions et ne restent pas de simples déclarations formelles.

Une compréhension aussi large de la question peut évidemment conduire à un chevauchement avec les deux autres thématiques retenues dans cette contribution, d'autant que le droit du travail est sous la pression constante du droit commercial et des postulats de l'économie libérale. Cette dernière est à l'origine d'un phénomène quasi universel de "fuite » ou "d'évitement » de la législation sociale si bien que son champ d'application, les notions de travail et de travailleur doivent être continuellement redéfinies afin de prendre en compte les nouveaux modes d'organisation des entreprises ${ }^{8}$. Cela signifie que la législation du travail devrait être presque entièrement renouvelée. Ceci étant dit, nous nous limiterons à examiner l'intervention publique dans les systèmes de relations de travail $(A)$, les procédures de règlement des conflits du travail, $(B)$ les difficultés d'application des normes juridiques internationales et supranationales $(C)$, enfin, l'efficacité de la négociation collective (D).

\section{A - L'INTERVENTION PUBLIQUE DANS LES RELATIONS DE TRAVAIL}

Les études sur la conception et le fonctionnement de l'inspection du travail sont diverses et très nombreuses dans le monde entier. Elles se concentrent soit sur des systèmes applicables dans des pays spécifiques, soit sur des analyses multi-pays utilisant des méthodes de droit comparé. Cette deuxième catégorie mérite d'être particulièrement mentionnée car la nature de ces études donne à leurs conclusions plus de portée et parce qu'elles permettent de mettre en évidence une diversité des fonctions assignées aux structures administratives ${ }^{9}$. Il est intéressant de noter que les inspecteurs du travail n'interviennent pas toujours exclusivement comme agents chargés de sanctionner les infractions légales en infligeant des amendes, mais qu'en outre ils conseillent et informent les entreprises par des recommandations, voire même en agissant comme médiateurs dans certains types de conflits $^{10}$.

8 Le soi-disant « co-working " (à ne pas confondre avec le « crowd-work ») est un bon exemple de cette pratique, il s'agit d'un nouveau concept associé au terme général "économie solidaire " ou « économie collaborative/de partage » bien que très différent de la plupart de ces concepts. G. Auzero, "Co-emploi », RDT, 2016-1, p. 27, Y. Pagnerre, "Critère du coemploi », RDT, 2016-3, p. 175. Pour une contribution très intéressante, voir la monographie spéciale BCLR, 2016, $n^{\circ} 94$, sur les «nouvelles formes d'emploi en Europe », qui analyse cette question dans plus de 38 pays.

9 T. Suzuki, "International Trends in Systems for Inspection of Labor Law Violations », JLR, 2016-4, vol. 13 (Special Edition: Illegal Labour), pp. 83, dans le même numéro, mais centré sur le droit interne, voir R. Yamakawa, "Policy measures to tackle violations of labor and employment laws in Japan », p. 98.

10 A. Vid. Fine, "Afterword: The Franco-Iberian Model from the U.S. Perspective », CLLPJ, 2016-2, 
D'autres articles soulignent toutefois que la numérisation et les progrès dus nouvelles technologies peuvent aussi aider à contrôler l'activité, y compris en matière de sécurité sociale, et à réduire l'informalité, caractéristique de certains systèmes de relations de travail. Un exemple est l'utilisation de dossiers électroniques et de bases de données qui ont été créés ex novo à cette fin ${ }^{11}$.

Une autre question connexe est celle du statut des lanceurs d'alerte, particulièrement lorsque ce sont des travailleurs. C'est un sujet pertinent non seulement parce qu'il nécessite une intervention publique mais aussi parce qu'il est basé sur le signalement anonyme, une technique traditionnelle de contrôle des systèmes. Les travailleurs doivent être protégés par une obligation appropriée de maintien du secret et de la confidentialité, ainsi que par la garantie du maintien de leur emploi ${ }^{12}$. La notion de lanceur d'alerte est apparue à l'origine comme un instrument dans divers domaines, comme la lutte contre la corruption et les crimes mettant gravement en danger la santé et la sécurité publiques. Elle devrait également avoir des effets significatifs sur les questions de travail, en particulier lorsque le plaignant est un travailleur du secteur privé pour qui la protection générale des témoins est souvent insuffisante et qui ne bénéficie pas des garanties accordées aux fonctionnaires ${ }^{13}$.

Ces conditions ont été établies dans l'un des derniers rapports du Groupe d'examen de la mise en œuvre (IRG) agissant au nom de la Conférence des États parties (COSP), qui a approuvé la Convention des Nations Unies contre la corruption (UNCAC), signée le 3 octobre 2017 par 140 pays, dont l'article 33 stipule que « Chaque Etat Partie envisage d'incorporer dans son système juridique interne des mesures appropriées pour assurer la protection contre tout traitement injustifié de toute personne qui signale aux autorités compétentes, de bonne foi et sur la base de soupçons raisonnables, tous faits concernant les infractions établies conformément à la présente Convention ».

Dans ce vaste domaine qu'est l'intervention publique, il convient de souligner la publication d'autres types de travaux, tout aussi intéressants, sur l'impact de la participation du secteur privé dans l'action publique. Cette étude comprend une série d'articles ${ }^{14}$ qui examinent le type et le degré de contrôle que les organismes publics peuvent exercer, non en tant qu'autorité mais en tant que partie contractante, selon le régime appliqué aux

vol. 3, p. 397, avec des contributions de l'Argentine, du Brésil, de la France, de l'Italie, du Québec et de l'Espagne.

11 Voir J. Bernardo Alvarado, "¿En el partidor? ", AL, 2016-472, vol. XL, p. 6. Pour un point de vue critique de SUNAFIL (Superintendencia Nacional de Fiscalización Laboral), dans le même numéro, voir A. Vilela, "Problemática actual en el cumplimiento de la función inspectiva », p. 12. J.A. N. Bernad, «Actas de liquidación fundadas en contravenciones de normativa extralaboral: evolución histórica y jurisprudencial »,RDS, 2016, n 74, p. 87.

12 H. Zimmermann, "Zur Problematik anonymer Arbeitnehmerbescwerden », AuR, 2016-6, vol. 64, p. 226. Du point de vue de la directive 2009/52/CE du Parlement européen et du Conseil du 18 juin 2009 prévoyant des normes minimales concernant les sanctions et les mesures à l'encontre des employeurs de ressortissants de pays tiers en séjour irrégulier, M. Giaconi, « Le politiche europee di contrasto al lavoro sommerso. Tra (molto) loi douce e (poco) loi dure», LD, 2016-3, p. 439. Sur cette directive et dans le même numéro, voir « Undeclared work e Unione europea, ultimo atto: una «piattaforma europea» per contrastare il lavoro sommerso, » p. 461.

13 Pour savoir si cette protection est efficace dans tous les pays, et particulièrement en Allemagne, voir A. Fischer-Lescano, "Internationalrechtliche Regulierung des Whistleblowing. Anpassungsbedarf im deutschen Recht (Teil1-2)», AuR, 2016-1/2, vol. 64, pp. 4 et 48.

14 Voir J. Cruz Villalón, "Claves laborales de la participación privada en la actuación pública », $T L$, $n^{\circ} 135 / 2016$, p. 13 
travailleurs des entreprises fournissant directement des services publics essentiels. On peut citer des exemples comme les services de santé ou l'éducation, ainsi que de nombreuses autres activités développées dans le cadre du secteur public dans son ensemble (nettoyage, entretien, sécurité, etc.). Ces articles examinent des questions relatives aux droits collectifs et aux droits à l'information, ainsi que sur le respect des normes de santé et de sécurité, mais aussi des questions plus spécifiques telles que les garanties de paiement effectif des salaires ${ }^{15}$ ou la légalité de l'emploi temporaire ${ }^{16}$.

\section{B - PROCÉDURES RELATIVES AUX CONFLITS DU TRAVAIL}

Nous ne tenterons pas d'être exhaustifs et ne présenterons pas l'ensemble des problèmes générés par les décisions judiciaires, en particulier celles de nature strictement formelle ou procédurale ${ }^{17}$. Nous laisserons également de côté les questions relatives aux systèmes de résolution extrajudiciaire des conflits même s'il existe des études et des réflexions théoriques intéressantes sur le rôle des juges dans une démocratie constitutionnelle en matière de droits collectifs, dans la mesure où ils deviennent un critère d'évaluation du système démocratique dans son ensemble ${ }^{18}$.

D’un point de vue complètement différent, la revue de littérature permet de relever des études statistiques sur la pratique judiciaire nationale ${ }^{19}$, très utiles pour mettre en lumière

15 C. Sáez Lara, « Garantías y responsabilidades en materia salarial», TL, n 135/2016, p. 345.

16 M. Rodríguez-Piñero Royo, "Gestión privada, contratación temporal y calidad en el empleo», $T L$, $n^{\circ} 135 / 2016$, p. 111

17 Voir N. Pogorelčnik Vogrinc, "Razlogi za obnovo postopka v individualnih delovnih sporih », $E$ \& $E, 2016-2 / 3$, vol. XVI, p. 283, au sujet d'une procédure de révision judiciaire extraordinaire permettant un examen ouvert de questions telles que la violation substantielle de la procédure, les infractions criminelles et les nouveaux faits et éléments de preuve nouveaux. Dans le même numéro, B. Kresal, "Obstoj delovnega razmerja kot predhodno vprašanje v delovnih sporih », traite des questions relatives à l'existence d'une relation de travail comme question préliminaire dans les conflits du travail, p. 217-240. C. Barnard, «Enforcement of Employment Rights by EU-8 Migrant Workers in Employment Tribunals», ILJ, 2016-1, vol. 45, p. 28 ; Voir aussi O. Fotinopoulou Basurko, "La inmunidad de jurisdicción y los contratos de trabajo en la LO 16/2015 ", RDS, $n^{\circ} 73 / 2016$, p. 69.

18 J.R. Paquette, "The Call for Deference in Labour Relations: An Answer to Justice Rothstein », CL\&ELJ, 2016-2, vol. 19, p. 101. Pour des commentaires intéressants sur le rôle d'un juge particulier (Antonin Scalia) dans le système américain, en particulier sur l'avenir des soi-disant " clauses d'atelier d'agence ", voir "Verso la fine del sindacato americano? La costituzionalità dell'obbligo di pagamento del contributo sindacale al vaglio della Corte Suprema degli Stati Uniti d'America », GDLRI, 2016-4, vol. 152, p. 759. Voir également, Szymanski, « Scalia's shadow: the Friedrichs Case and the fate of the American labor movement », même édition, p. 761. Pour en savoir plus sur le rôle des juges, voir G. Golding, "The Role of Judges in the Regulation of Australian Employment ", IJCLLIR, 2016-1, vol. 32, p. 69. Voir également, M. Nogueira Guastavino, " La libertad sindical negativa en Estados Unidos », DRL, 2016-4, p. 374.

19 Pour les données et les analyses au Japon et en Allemagne, voir K. Hamaguchi, "Comparative Analysis of Employment Dispute Cases Resolved by Labor Bureau Conciliation, Labor Tribunals and Court Settlement », JLR, 2016-2, vol. 13, p. 119, et G. Grotmann Höfling, " 20 years of labour courts' joint statistics - an examination of basic points ", AuR, 2016-11, vol. 64, p. 407. Du même auteur et dans la même revue, voir " Die Arbeitsgerichchtsbarkeit 2015 im Lichte der Statistik», 2016-12, p. 497. II montre que le temps moyen de résolution des litiges est inférieur à un an, les appels ont diminué et sont le plus souvent ignorés (55\%), et les procédures à l'initiative des employeurs ont augmenté. II souligne également que le processus juridique est généralement traité par des avocats et rarement par des comités d'entreprise ou directement par des salariés. 
la réalisation des principaux objectifs assignés aux systèmes procéduraux. Elles permettent d'évaluer, par exemple, la durée moyenne des procédures, l'activité de chaque type de tribunal (volume de travail et sens des décisions), le profil des plaignants, et, bien sûr, les questions ou types d'affaires faisant l'objet de poursuites.

Une autre catégorie d'analyses pertinentes porte sur l'existence, voire la critique de réformes mises en œuvre. Parmi ces travaux, des articles consacrés à l'impact de l'adoption au Royaume-Uni de diverses mesures d'austérité et du Trade Union Act $2016^{20}$ ont retenu notre attention. Une des critiques faites à cette loi est qu'elle a entraîné l'imposition de frais judiciaires dans ce type de procédures, mais surtout que les employeurs ont pu de nouveau avoir recours à la vaste gamme des ressources offertes par à la Common law y compris au concept de responsabilité des dommages (délit) pour faire face à des grèves ou à d'autres actions collectives ${ }^{21}$.

Notre dernière attention dans ce chapitre doit sans aucun doute porter sur la première des catégories juridiques celle des droits fondamentaux des personnes. Le fait que les Déclarations ou les Chartes élèvent de nombreuses garanties essentielles du travailleur pour les inclure dans cette catégorie afin de leur assurer une application effective découle sans aucun doute de la constitutionnalisation d'une grande partie du droit du travail22. Cela signifie que toutes les techniques d'approche et d'interprétation des normes constitutionnelles s'appliquent, y compris l'équité, la suspension préventive des effets, les règles d'inversion de la charge de la preuve et la nullité des actes ${ }^{23}$. La liste des références deviendrait très longue si nous incluions toutes les protections anti discrimination, en raison de motifs prohibés (sexe ${ }^{24}$, race, religion, nationalité, âge, handicap ${ }^{25}$, etc.), ou en raison d'un type d'emploi particulier, sachant que la plupart des cas sont liés à un licenciement ${ }^{26}$.

20 Pour l'analyse d'un cas similaire en France, voir D. Marshall, and M. Henry, "Vers une normalisation de la justice prud'homale ? », RDT, 2016-7/8, p. 457. Voir également, Ph. Martin, « La reforma laboral en Francia », TL, n 134/2016, p. 45.

21 A. Bogg, "Beyond Neo-Liberalism: The Trade Union Act 2016 and the Authoritarian State ", ILJ, 2016-3, vol. 45, p. 299. Pour une vue générale de la question, voir « Book Review: New Frontiers in Empirical Labour Law Research », ILJ, 2016-2, vol. 45, p. 263. Voir également, D. Cabrelli, "Liability and Remedies for Breach of the Contract of Employment at Common Law: Some Recent Developments », ILJ, 2016-2, vol. 45, p. 207.

22 F. Valdés Dal-Ré, "Constitucionalismo laboral europeo y protección multinivel de los derechos laborales ", DRL, 2016-2, p. 91. Du même auteur dans la même revue (co-editeur), voir " Las libertades de reunión y de asociación en el CEDH (ECHR)», DRL, 2016-4, p. 301.

23 M.V.Ballestrero, "Tra discriminazione e motivo illecito: il percorso accidentato della reintegrazione», DLRI, 2016-2, vol. 38, p. 231.

24 A. Heron, "Effective protection of pregnant women at work: Still waiting for delivery? ", $A J L L$, 2016-1, vol. 29, p. 24.

25 S. Varva, "Promozione dei soggetti svantaggiati negli appalti pubblici. La regolazione locale e la direttiva 2014/24/UE », LD, 2016-1, p. 53.

26 M. Benecke, "AGG und Kündigungsschutz - da BAG und die diskriminierende Kúndigung ", AuR, 2016-1, vol. 64, p. 9, discutant du rôle de la Cour fédérale du travail sur les licenciements discriminatoires. 


\section{C - APPLICATION DES RÉGLEMENTATIONS INTERNATIONALES ET SUPRANATIONALES}

Cette question est étroitement liée au développement qui précède car les normes internationales et supranationales fournissent à tous les systèmes étatiques de nouvelles listes de droits de valeur supérieure, dotés du statut de droits de l'homme ou de droits sociaux fondamentaux. La littérature révèle de profondes difficultés de coordination dans l'application de constructions au niveau mondial ou international compatibles avec celles de chaque ordre juridique interne. Ces problèmes sont aussi parfois liés aux formulations délibérément floues ou trop générales des normes, d'où l'importance des programmes d'amélioration technique des réglementations telles que REFIT ${ }^{27}$.

Une autre conséquence réside bien entendu dans le fait que de nombreux organismes nationaux continuent d'émettre des réserves lorsqu'ils sont contraints de modifier quelque peu leurs constructions établies, agissant comme des gardiens zélés de l'idée de la souveraineté de l'Etat ${ }^{28}$.

Ce domaine s'est considérablement développé ces dernières années parce que les acteurs ont besoin de nouvelles sources ou ressources pour fonder leurs revendications, en particulier lorsqu'ils constatent que leurs demandes sont rejetées au niveau national, du fait de la jurisprudence, ou en raison de l'impact des réformes juridiques. Cela les conduit à rechercher une nouvelle légitimité dans les réglementations internationales ${ }^{29}$.

Pour des raisons évidentes, le droit social européen ou de l'Union européenne est le sujet d'un grand nombre d'articles. Ce n'est pas seulement parce que ce droit est l'objet même des revues scientifiques mais c'est aussi parce qu'il traite de questions relatives à la législation sur la sécurité sociale ou à la protection sociale. S'ajoutent à cela d'autres explications. La nature supranationale des réglementations de l'UE se reflète également dans la défense constante par la CJUE de ses propres concepts. II appartient donc aux systèmes nationaux de s'y adapter et non l'inverse ${ }^{30}$. Cela en fait une source constante de nuances et de nouveaux alignements sur presque toutes les questions sociales.

27 Le programme «Réglementation, aptitude et performance réglementaires » (REFIT) de la Commission veille à ce que la législation de l'UE produise des résultats pour les citoyens et les entreprises de manière efficace, efficiente et à moindre coût. REFIT vise à simplifier la législation de I'UE, à supprimer les charges inutiles et à adapter la législation existante sans compromettre les objectifs politiques. Voir I. Schömann, « Mieux légiférer dans l'Union Européenne : simplifier ou dénaturer l'acquis communautaire? Analyse critique d'une initiative de la Commission au regard du Droit du travail », RDCTSS, 2016-1, p. 6.

28 F. Villanueva, "La définition du niveau de négociation collective au Pérou : un processus singulier d'appropriation des décisions du Comité de la liberté syndicale de l'OIT par le Tribunal constitutionnel », RDCTSS, 2016-1, p. 70. G. Anderson, "The Common Law and the Reconstruction of Employment Relationships in New Zealand », IJCLLIR, 2016-1, vol. 32, p. 93.

29 J. Cabeza Pereiro, "Algunas reflexiones a la vista del asunto TYCO sobre el concepto "tiempo de trabajo" », DRL, 2016-1, p. 36.

30 B. Creighton, "Who Is a "Worker" in International Law?», CLLPJ, 2016-3, vol. 37, p. 691. 
Les questions abordées sont diverses et présentent un grand intérêt. II s'agit notamment : des migrations internationales ${ }^{31}$, du détachement de travailleurs dans le cadre de la prestation de services ${ }^{32}$, des contrats temporaires ${ }^{33}$, les conséquences autres que la nationalité de l'étranger ${ }^{34}$, sans parler des autres sujets décrits ci-dessus ou qui seront traités dans les sections suivantes.

\section{D - EFFICACITÉ DE LA NÉGOCIATION COLLECTIVE}

Comme nous l'avons souligné dès le départ, lorsqu'on se réfère à l'efficacité des normes du travail, on ne peut éviter de mentionner l'aspect le plus caractéristique de toute la législation du travail, à savoir la reconnaissance des conventions collectives comme principale source de réglementation des conditions de travail. Cependant, l'existence de véritables conventions collectives dépend évidemment de quelque chose de plus que la simple reconnaissance de cet instrument formellement distinct ${ }^{35}$. L'autonomie collective authentique dépend de l'existence de la liberté d'association et du droit à l'action collective, et en particulier du droit de grève. Deux axes principaux, unis par des critères communs évoqués précédemment, permettent d'illustrer nos principales préoccupations en la matière. Cela ne doit pas impliquer d'oublier pour autant le rôle de l'autonomie individuelle ${ }^{36}$.

En premier lieu, nous nous référons à l'ensemble des réformes menées dans certains pays dans la mesure où elles révèlent des tendances importantes. C'est le cas, par exemple, en Espagne, où l'impact de certains changements majeurs introduits en 2012, sur l'efficacité des conventions collectives, concernant l'intangibilité du contenu de l'accord et son application scrupuleuse, est encore à l'étude. Cela revient à interdire les modifications par des sujets autres que les négociateurs eux-mêmes. La nouvelle loi a maintenant permis cela en établissant un arbitrage obligatoire pour les parties en cas de crise dans l'entreprise. Dans ces situations, la sentence arbitrale fixe de nouvelles conditions de travail temporaires applicables ${ }^{37}$. Quelque chose de similaire s'est produit en Italie lors des réformes de 2015, quoique dans une moindre mesure. Cela signifie que la mise en œuvre d'une convention collective d'entreprise est prioritaire par rapport à une convention collective nationale, du moins dans certains domaines. La structure de la négociation et, en particulier, l'équilibre des pouvoirs sont donc modifiés de manière significative, non seulement pour les

31 M. Ruhs, "The rights of migrant workers: Economics, politics and ethics », ILR, 2016-2, vol. 155, p. 283.

32 M. Rodríguez-Piñero y Bravo-Ferrer, «El desplazamiento temporal de trabajadores y la Directiva 2014/67 relativa a la garantía de cumplimiento de la Directiva 96/71/CEE », DRL, 2016-5, p. 407.

33 EuZA, 2016-2, Special topic «Temporary Work», for all: Junker, A., « Arbeitnehmerüberlassung und Werkverträge - Der europäische Rahmen », p. 141.

34 L. Campling, J. Harrison, B. Richardson and A. Smith, "Can labour provisions work beyond the border? Evaluating the effects of EU free trade agreements », ILR, 2016-2, vol. 155, p. 357.

35 Parfois, les domaines semblent se recouper, C. Graeme, "Litigation as an alternative to collective bargaining ", about "sleepover " cases in New Zealand, BCLR, 2016, n 92, p. 217. H. Cialti, "Solución a la pérdida de ultraactividad del convenio sin nuevo acuerdo: El caso francés », DRL, 2016-2, p. 173.

36 A. Jeammaud, "Contrat individuel de travail et accord collectif de travail », RDT, 2016-4, p. 228.

37 N. Mendoza Navas, « Los procedimientos de inaplicación de condiciones de trabajo al amparo de las decisiones de la Comisión Nacional Consultiva de Convenios Colectivos », RDS, n 74/2016, p. 61. 
différentes organisations représentatives des travailleurs mais dans le cadre de sociétés spécifiques. En effet, à un niveau territorial aussi circonscrit, les possibilités pour les entrepreneurs d'exercer leur influence de manière beaucoup plus décisive se multiplient de façon exponentielle ${ }^{38}$.

En ce qui concerne la liberté d'association et, plus particulièrement, le droit de grève, de nombreux articles portent sur les droits fondamentaux d'action et de conflit collectif ${ }^{39}$. Indiscutablement, cette question constitue le sujet principal des articles, soit parce que des réformes juridiques importantes ont eu lieu (Royaume-Uni) ${ }^{40}$, soit à cause du rôle décisif des nouvelles décisions judiciaires (Canada) ${ }^{41}$. Plus précisément, la loi impose certaines conditions pour que les appels à l'action collective ou les revendications soient légitimes et proportionnées, principalement par des bulletins de vote pré-grève de tous les travailleurs concernés et en fixant des limites pour garantir l'efficacité des autres droits pour le reste des citoyens.

\section{|| - LES DIFFICULTÉS PRÉSENTÉES PAR LA NATURE CHANGEANTE DE L'EMPLOI}

Plus de 100 articles publiés dans les revues membres en 2016 ont traité des défis de la réglementation du travail lorsque la nature de l'emploi évolue de façon aussi rapide. De manière générale, les articles portaient sur deux questions. Premièrement, que se passe-t-il réellement sur le marché du travail ? Comment évolue la nature de l'emploi et pourquoi? Et deuxièmement, quels changements faut-il apporter à la réglementation du travail pour assurer une protection adéquate des travailleurs face à ces changements?

38 G.P. Cella, «Quale futuro per la contrattazione collettiva?», DLRI, 2016-2, vol. 38, p. 217.

39 M. Sädevirta, "Freedom of Association and the Right to Strike », EuZA, 2016-1, p. 445.

40 S. Moore, "We Planned a Dispute by Blackberry": The Implications of the Trade Union Bill for Union Use of Social Media as Suggested by the BA-BASSA Dispute of 2009 », ILJ, 2016, vol. 45, p. 251. Dans le même journal, voir R. Dukes, «Pre-strike Ballots, Picketing and Protest: Banning Industrial Action by the Back Door? », n³, p. 337.

41 R.J. Adams, «Bringing Canada's Wagner Act Regime into Compliance with International Human Rights Law and the Charter ", CLELJ, 2016-2, vol. 19, p. 53. Dans le même journal et le même volume, voir B. Etherington, "The Right To Strike under the Charter after Saskatchewan Federation of Labour: Applying the New Standard to Existing Regulation of Strike Activity », 2016-2, p. 117 et P. Cavalluzzo, "The Impact of Saskatchewan Federation of Labour on Future Constitutional Challenges to Restrictions on the Right To Strike », CLELJ, 2016-2, vol. 19, p. 117. Pour des comptes rendus dans d'autres pays, voir D. Peetz, "Industrial Action, The Right to Strike, Ballots and the Fair Work Act in International Context ", AJLL, 2016-2, vol. 29, p. 133, même revue et volume, B. Creighton, "Strike Ballots and the Law in Australia », p. 154 ; M. Wilson, "Strike Ballots: The New Zealand Experience », p. 194, P. Benjamin, «Strike Ballots in South Africa », p. 210. 


\section{A - COMMENT LE MARCHÉ DU TRAVAIL CHANGE-T-IL?}

La littérature reconnaît depuis des décennies que le modèle standard de l'emploi est en crise. Bien que ce modèle soit décrit de diverses façons, il correspond à un travail occupé par un salarié à temps plein et pour une durée indéterminée dans une même entreprise. Le salarié est souvent syndiqué, généralement un homme, rémunéré équitablement, jouissant d'une certaine sécurité de l'emploi et d'une gamme complexe de protections de l'emploi. Le problème central analysé par la littérature porte sur le cadre de la protection juridique des travailleurs élaboré à partir du modèle standard. Or, la nature du travail a changé ${ }^{42}$.

La doctrine en 2016 a été principalement intéressée par la grande variété des modèles d'emploi qui coexistent avec le modèle standard. Les changements affectent autant l'employeur que les salariés ${ }^{43}$.

Du côté de l'employeur, tout d'abord, on observe une «fissuration ». Cette idée formulée et décrite par David Weil ${ }^{44}$, permet de souligner que par le passé, un seul employeur unique pouvait employer tous les travailleurs d'un site donné, alors que maintenant plusieurs employeurs peuvent intervenir. Un exemple classique est celui d'un hôtel où, autrefois, presque tous les travailleurs d'un hôtel dont l'enseigne indiquait " Hyatt » sur la porte d'entrée étaient des salariés de Hyatt, tandis qu'aujourd'hui une grande partie du travail de I'hôtel peut être sous-traitée à des employeurs divers. Le restaurant peut être exploité par un employeur différent, les services de nettoyage par un autre employeur, le système de réservation par un troisième employeur, etc.

Ensuite, «l'employeur » d'un travailleur peut ne pas être le destinataire et le bénéficiaire du travail du salarié, mais être un employeur intermédiaire qui embauche le salarié et conclut un contrat avec l'employeur destinataire pour lui fournir (mettre à disposition) le travailleur. Avec quelques variantes ici et là, ce type d'arrangement contractuel est dénommé de diverses manières, travail intérimaire ${ }^{45}$, leasing de personnel (en Allemagne) ${ }^{46}$, payrolling (aux Pays-Bas) ${ }^{47}$, et portage salarial (en France) ${ }^{48}$. Mais comme la fissuration, il introduit la possibilité d'avoir une relation de travail multipartite plutôt que bipartite.

42 II convient de noter que les articles publiés dans les revues spécialisées portaient sur la réglementation du travail dans les économies développées, où l'on se préoccupe de la baisse des normes et des protections du travail. C'est donc aussi l'objet de cette étude. Les nouvelles formes de travail sont toutefois très présentes dans les pays en développement et les problèmes peuvent être très différents, surtout si les nouvelles technologies permettent des gains rapides de productivité du travail. Voir E. Wamboye, A. Adekola \& B. Sergi, «ICTs and Labour Productivity Growth in Sub-Saharan Africa », ILR, 2016, vol. 155, p. 231.

43 Puisque nous parlons d'une relation, ces catégories ont tendance à se fondre les unes dans les autres, mais il est utile de les considérer séparément.

44 D. Weil, «The fissured workplace: why work became so bad for so many and what can be done to improve it », 2014.

45 V. de Stefano, "Introduction: Crowdsourcing, the Gig-Economy, and the Law », CLLPJ, 2016, vol. 37 , p. 461.

46 W. Hamann, "The Draft Bill on the Modification of the Personnel Leasing Act and Other Acts ", AuR, $2016, n^{\circ} 4$.

47 J. P.H. Zwemmer, "Payrolling in the Netherlands », BCLR, 2016, vol. 94, p. 117.

48 Fr. Kessler, " "Employees Without a Boss": Portage Salarial in France », BCLR, 2016, vol. 94, p. 103. 
Par ailleurs, une catégorie émergente "d'employeurs » affirme qu'ils ne sont pas du tout des employeurs, mais plutôt des plates-formes logicielles qui mettent seulement en relation des travailleurs disponibles avec des personnes disposées à payer les services offerts. Uber et Lyft, des entreprises importantes qui connectent des chauffeurs et des personnes ayant besoin d'être transportées en sont de parfaits exemples. On pourrait considérer cela comme un autre type d'entente tripartite, sauf qu'en l'espèce aucune des parties n'est disposée à accepter le rôle de l'employeur. Il existe d'autres évolutions du côté de l'employeur, mais celles susmentionnées devraient suffire à illustrer la nature et la portée des changements.

De la même manière, des changements spectaculaires peuvent être observés du côté des salariés. L'un d'eux est que les salariés travaillent de plus en plus souvent hors des locaux de l'employeur. Cela n'est pas nouveau naturellement, si on prend par exemple le travail à domicile et le télétravail. Mais les technologies modernes de l'information et de la communication (TIC) ont accru leur incidence et créent de nouveaux problèmes. Par exemple, la question de la qualification de temps de travail est devenue plus complexe car les TIC permettent aux employeurs de contacter les travailleurs n'importe où et n'importe quand, et d'attendre des réponses rapides.

Un autre changement réside dans le fait que les liens entre les travailleurs et les employeurs sont souvent plus ténus. L'exemple typique est le contrat zéro heure au Royaume-Uni, par lequel les employeurs acceptent de rémunérer le travail accompli, mais ne s'engage pas à fournir une quantité ni précise ni minimale de travail. Ces contrats sont sans doute d'une grande utilité pour les employeurs pour répondre à l'évolution de la demande de main-d'œuvre, mais ils augmentent l'insécurité des travailleurs et relâchent le lien entre l'employeur et le salarié.

Les TIC permettent à ces liens de devenir encore plus lâches. Le crowdfunding est une sorte de contrat zéro heure poussé à l'extrême. Les employeurs offrent des tâches d'ampleur très variable (mais généralement de très petites tâches) sur une plate-forme électronique à laquelle ont accès des travailleurs désireux d'effectuer ces tâches. Les travailleurs sont souvent encouragés à se faire concurrence (parfois cela est présenté sous forme de jeu) et ils sont généralement très peu payés pour chaque tâche. On peut considérer cela comme une sorte de contrat zéro heure avec des liens encore plus faibles entre l'employeur et le travailleur. Du côté des employeurs, encore une fois, personne dans cet arrangement n'est prêt à assumer l'étiquette "d'employeur » ${ }^{49}$.

Enfin, la nature même du travail évolue rapidement. Une proportion croissante du travail appartient au nouveau format de la " gig economy " ou économie des petits boulots : Nommée ainsi à partir de représentations du travail pour les musiciens, la " gig economy » suppose généralement que les artistes apportent leur propre équipement, déterminent leurs horaires et le nombre d'heures et fournissent un service directement au consommateur tandis qu'un intermédiaire tiers promeut le service, fournit la plate-forme à travers laquelle l'interprète et le consommateur interagissent et perçoit le paiement du consommateur, en retenant un pourcentage pour lui-même et en transmettant le reste à l'interprète. L'interprète a besoin de relations, de la commodité et de la reconnaissance que l'intermédiaire lui fournit; l'intermédiaire a besoin de l'interprète pour fournir le service. C'est cette relation d'interdépendance qui distingue les relations de travail dans la

49 Voir W Daubler, Th. Klebe, « Crowdwork: datore di lavoro in fuga?», DLRI, 2016, vol. 151, p. 471. 
" gig economy " des relations de travail standard des salariés ou des travailleurs indépendants ${ }^{50}$.

Cette forme de travail se développe rapidement pour deux raisons principales : une proportion de plus en plus importante du travail est uniquement cognitive et en raison de l'informatisation, elle peut être faite n'importe où et n'importe quand. Une caractéristique unique et quelque peu troublante de ce type de travail est qu'il peut se réaliser (et c'est le cas généralement) sans aucune interaction humaine directe, l'employeur comme le salarié $\mathrm{n}^{\prime}$ interagissant plus qu'avec leurs ordinateurs ${ }^{51}$.

Comme nous l'avons indiqué, ces changements et la façon dont le droit du travail et de l'emploi devrait y répondre a fait partie des sujets les plus discutés dans les revues membres de I'IALLJ en 2016. Mais quelle est la prévalence de ces changements sur la nature de l'emploi ? D'un côté, la proportion de travailleurs engagés dans ce type de régimes de travail alternatifs reste encore beaucoup plus faible que celle de travailleurs engagés dans des relations de travail plus traditionnelles. Une étude récente aux Etats-Unis a révélé par exemple que le pourcentage global de travailleurs engagés dans l'un ou l'autre type de relation de travail alternative était de 15,8\%. Plus précisément, l'étude a révélé que $3,1 \%$ des travailleurs étaient embauchés par des entreprises sous contrat et que seulement $0,5 \%$ des travailleurs fournissaient des services par le biais d'intermédiaires en ligne (comme Uber ou Amazon Mechanical Turk). Cela donne l'impression que l'accent mis par les revues sur les autres formes de travail et en particulier le haut niveau d'attention accordé à l'emploi par intermédiation n'est pas entièrement justifié. D'autre part, l'étude a révélé qu'une proportion étonnante de $94 \%$ de la croissance nette de l'emploi aux Etats-Unis de 2005 à 2015 concernait d'autres formes de relation de travail ${ }^{52}$. Si les formes d'emploi qui représentent l'avenir ne sont pas encore complètement en place, elles se propagent rapidement. Peut-être, les revues académiques ont-elles simplement une certaine avance sur la tendance?

\section{B - COMMENT LA RÉGLEMENTATION DU TRAVAIL DEVRAIT-ELLE S'ADAPTER AUX CHANGEMENTS DU MARCHÉ DU TRAVAIL?}

Les articles publiés dans les revues au cours de l'année 2016 portaient sur les options retenues par les Etats pour repenser la réglementation du travail afin de faire face aux changements en matière l'emploi.

Un des domaines d'étude privilégié est celui de la classification des travailleurs qui ne relèvent pas du modèle standard d'emploi. Traditionnellement, on distingue les salariés des travailleurs indépendants. L'une des questions porte sur le fait que les employeurs structurent l'emploi de manière à éviter la protection conférée par le droit du travail et de l'emploi ou, pire, oeuvrent intentionnellement pour éviter l'application de leurs obligations. En ce qui concerne les erreurs de qualification de la relation de travail, plusieurs articles

50 S. L.Willborn et al., Employment law: cases and materiales, 38, 2017.

51 Pour un bon aperçu, voir L. Tičar, «The Impact of Digitalisation on the Emergence of New Forms of Work », E\&E, 2016, vol. 16, p. 241.

52 L. F. Katz, A. B. Krueger, "The Rise and Nature of Alternative Work Arrangements in the United States, 1995-2015», Nber Working paper series, 2016, n²2667. 
ont porté sur le faux travail indépendant ${ }^{53}$ et sur l'emploi « dissimulé ${ }^{54}$, notamment en ce qui concerne les nouvelles formes d'emploi. Mais le problème le plus profond soulevé par nombre de ces nouvelles formes d'emploi n'est pas tant une mauvaise qualification que le fait que les travailleurs ne relèvent pas à proprement parler de l'une ou l'autre catégorie. Le problème a été bien expliqué par un juge américain qui, lorsqu'il s'efforçait de déterminer si les chauffeurs de Lyft étaient des salariés, s'est exprimé de manière imagée et estimait que c'est comme si le jury « recevait une cheville carrée et qu'on lui demandait de choisir entre deux trous ronds ${ }^{55}$. Les critères mis au point par les tribunaux après des décennies pour identifier les salariés et les distinguer des autres catégories de travailleurs ne s'appliquent tout simplement pas très facilement à un emploi fissuré ou à de nombreuses formes de travail liées aux $\mathrm{TIC}^{56}$. Les tribunaux ont du mal à classer ces formes de travail soit comme travail salarié soit comme travail indépendant lorsque ni l'une ni l'autre de ces catégories ne semble convenir réellement.

Une autre approche consiste à créer un «trou carré » pour cette cheville inhabituelle, qu'est cette nouvelle catégorie de travailleurs émergents. Le travailleur appartenant à cette catégorie ne serait pas tout à fait un salarié ni tout à fait un travailleur indépendant, mais se situerait entre les deux. Au lieu d'un travailleur indépendant, le travailleur appartenant à cette nouvelle catégorie peut être considéré comme un travailleur dépendant. Cette catégorie aurait droit à certaines des protections de travail offertes aux salariés, pas à l'ensemble des protections. Ainsi, une proposition émise aux Etats-Unis protégerait cette catégorie de travailleurs contre les discriminations et leur fournirait une assurance contre les accidents de travail, mais n'imposerait pas qu'on leur verse un salaire minimum ou qu'ils obtiennent une assurance maladie ${ }^{57}$. A quelques différences près, cette approche générale a été mise en œuvre dans plusieurs pays sous des appellations différentes, telles que, autoentrepreneur (en France) ${ }^{58}$, para-subordonné (en Italie) $)^{59}$, salariés économiquement dépendants (en Hongrie) ${ }^{60}$, et travailleurs autonomes économiquement dépendants (en Espagne $)^{61}$. S'il est vrai que cette approche se répand de plus en plus, elle ne fait cependant

53 T. Gyulavári, « Civil Law Contracts in Hungary », BCLR, 2016, vol. 94, p. 79, 81, J. J. Abrantes, R. Canas da Silva, « New Forms of Employment in Portugal », BCLR, 2016, vol. 94, p. 313-14.

54 G.Tavits, « New Forms of Employment in Estonia», BCLR, 2016, vol. 94, p. 193, 196-97.

55 Cotter v. Lyft, Inc., 60 F. Supp. 3d 1067, 1081 (N.D. Cal. 2015).

56 Jeremias Prassl a publié un livre important en 2015 décrivant bien les problèmes d'identification des salariés et proposant une solution centrée sur les employeurs. The Concept of the Employer. Le livre a été référencé dans des revues membres en 2016. Voir par exemple, J. Fudge, "The Concept of the Employer ", ILJ, 2016, vol. 45, p. 270, L. Ratti, "Intorno al-i concetto-i di datore di lavoro. A proposito di The Concept of the Employer di Jeremias Prassl », DLRI, 2016, vol. 150, p. 381.

57 S. Harris, A. Kruger, A Proposal for Modernizing Labor Law for Twenty-First-Century Work: The "Independent Worker », The Hamilton Project, 2015.

58 J.-E. Ray, J. Rojot, «The Fissured Workplace in France », CLLPJ, 2016, vol. 37, p. 463.

59 B. Creighton, Sh. McCrystal, "Who is a "Worker" in International Law? », CLLPJ, 2016, vol. 37, p. 691.

60 T. Gyulavári, supra note 53 , at 87.

61 J. García Murcia, Iván Rodríguez Cardo, "New Forms of Employment in Spain », BCLR, 2016, vol. 94, p. 349, 352-53. 
pas l'unanimité. Valerio de Stefano, par exemple, pense qu'il est trop difficile de définir le type de travailleurs qui entrerait dans la catégorie de la protection limitée et que cette catégorie peut constituer un obstacle à la pleine protection du travail ${ }^{62}$.

Les deux premières façons de traiter les nouvelles formes de travail tentent de refondre les règles générales qui peuvent s'appliquer à l'ensemble des grandes catégories de relations de travail. Une troisième approche consiste à identifier des relations d'emploi particulières qui posent des problèmes nouveaux et spéciaux et à développer des protections spéciales pour l'emploi dans ces catégories relativement étroites. Un exemple émergent de ceci réside dans l'utilisation de "bons». Ce système, utilisé dans plusieurs pays, permet à un " employeur » d'acheter un «bon » pour payer un travailleur. Le "bon " comprend le paiement des cotisations sociales, telles que celle de sécurité sociale. Le système n'est généralement disponible que pour certains types de professions (comme les travailleurs à domicile) qui, pour diverses raisons, ont tendance à fonctionner en dehors du système d'emploi normal en l'absence de bons ${ }^{63}$. Une autre approche consiste à traiter des problèmes individuels distincts présentés par de nouvelles formes de travail. Par exemple, les TIC permettent aux employeurs de solliciter les salariés à tout moment, ce qui augmente le nombre de problèmes liés au temps de garde des enfants. De même, étant donné que les TIC permettent aux personnes de travailler n'importe où et n'importe quand, il est également plus difficile de comptabiliser le temps effectivement travaillé. Aucun de ces problèmes susmentionnés n'est nouveau, mais les TIC augmentent leur incidence et leur importance. Pour y remédier, de nombreux pays ont repensé leurs règles ${ }^{64}$, la France étant la plus avancée à cet égard avec sa récente loi sur le «droit à la déconnexion ${ }^{65}$.

D'un autre côté, des articles traitent également de ce qu'il faut faire avec un employeur fissuré ou en voie de disparition. Le problème central est de savoir comment tenir les employeurs responsables du respect des obligations du droit du travail lorsque leurs relations avec les travailleurs sont lointaines et fragiles. Un certain nombre d'articles ont discuté des façons dont les lois s'ajustent pour régler ce problème. Une option consiste à élargir le concept d'emploi conjoint afin que plus d'un employeur puisse être responsable de la conformité aux lois lorsque plusieurs sont impliqués dans la relation de travailb6. Au lieu d'un emploi conjoint qui rend tous les employeurs responsables de tous les aspects liés à l'emploi, une autre approche consisterait à attribuer des responsabilités à des employeurs donnés, en fonction de leur relation particulière avec le travailleur. Ainsi, l'employeur qui embauche peut être responsable des questions relatives à l'embauche, celui qui gère le salarié au jour le jour pourrait être responsable des conditions sur le lieu de travail, etc. ${ }^{67}$.

62 V. de Stefano, "The Rise of the "Just-in-Time Workforce": On-Demand Work, Crowdwork, and Labor Protection in the "Gig-Economy" ", CLLPJ, 2016, vol. 37, p. 471, 494-99.

63 Plusieurs pays d'Europe ont mis en place des systèmes de bons. Symposium, « New Forms of Employment in Europe », BCLR, 2016, vol. 94, p. 1, 145 (Austria), 154 (Belgium), 231 (Greece), 345-46 (Slovenia), 364 (Turkey).

64 Voir B. Waas, "New Forms of Employment in Germany », BCLR, 2016, vol. 94, p. 223, 224-25.

65 Ch. Mathieu et al., « Droit á la Déconnexion, Santé au Travail », 2016, RDT, n Oct.

66 Voir D. Izzi, « Joint \& Several Liability for the Protection of Outsourced Work: Food for Thought from European Union Law», LD, 2016, vol. winter, p. 35.

67 Voir J. Prassl, « Uber, Taskrabbit, and Co.: Platforms as Employers? Rethinking the Analysis of Crowdwork », CLLPJ, 2016, vol. 37, p. 619. 
Une question primordiale dans la réponse juridique aux nouvelles formes d'emploi est de savoir s'il s'agit de problèmes vraiment nouveaux et uniques, ou s'il s'agit de problèmes évolutifs que la réglementation du travail a toujours dû affronter. Matthew Finkin soutient que, bien que nous puissions tirer une leçon des expériences passées, les problèmes de réglementation sont "véritablement nouveaux " parce que l'informatisation combinée à la nature cognitive du travail transforme les marchés locaux en marchés mondiaux, qui sont beaucoup plus difficiles à réglementer ${ }^{68}$. C'est possible. Peut-être y a-t-il eu d'autres périodes au cours desquelles les changements rapides et la mondialisation ont posé des défis particuliers à la réglementation du travail et nous devons donc concentrer notre attention sur les leçons de ces époques ${ }^{69}$. Mais qu'ils soient nouveaux ou anciens, il ne fait aucun doute que les défis lancés à la réglementation du travail sont complexes et difficiles.

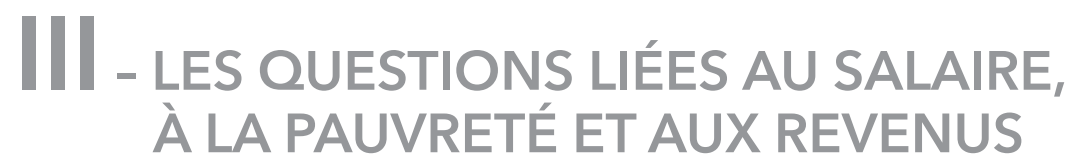

Même si la question de la pauvreté est loin d'être nouvelle pour les chercheurs en droit du travail et en droit de la sécurité sociale, il convient de souligner l'importance qui lui a été accordée par les auteurs de l'IALLJ en 2016. Nous souhaitons souligner deux perspectives qui ont particulièrement intéressé les auteurs sur la question de la pauvreté : les différents types et (relativement) nouveaux travailleurs précaires et les salaires. Ces travaux se situent en partie dans le prolongement de ceux des années précédentes à propos des conséquences de la crise financière et économique de 2008 et des réformes du droit du travail et du système de sécurité sociale en réponse à la crise ${ }^{70}$. D'autres facteurs ont donné lieu à des travaux sur la pauvreté et les salaires, tels que l'augmentation des activités à la frontière entre travail rémunéré et autres activités, ainsi que l'introduction d'un salaire minimum légal en Allemagne, l'idée d'un salaire minimum européen, et les débats croissants et intensifs sur «l'approche capacitaire ». Examinons les travaux sur la précarité du travail $(A)$, les salaires $(B)$, les régimes de protection sociale $C$ ), et enfin l'allocation de revenu de base (D).

68 "Beclouded work, Beclouded Workers in Historical Perspective », CLLPJ, 2015, vol. 37, p. 603, 617-18.

69 Voir par exemple D.T. Rod Rodgers, Atlantic Crossings: Social Politics in a Progressive age (1998) (passant en revue les mouvements sociaux des années 1870 aux années 1940, en réponse à la mondialisation accrue et aux changements technologiques rapides), J.F. Witt, The Accidental Republic: Crippled Workmen, Destitute Widows, And The Remaking Of American Law, 2004, (examinant les origines des systèmes d'indemnisation des travailleurs aux Etats-Unis, étant donné que les chemins de fer ont modifié la géographie et la technologie de la réglementation juridique des blessures accidentelles). Voir P. Končar, Digitalisation - The Challenges of Labour Law, E\&E, 2016, vol. 16, p. 257.

70 Sur les conséquences de la crise financière et économique, il convient de noter qu'un auteur italien discute d'une nouvelle tendance plutôt positive du point de vue de la protection des droits des travailleurs - un revirement de la part des juges constitutionnels italiens passant d'une approche d'urgence pour restreindre les droits sociaux des travailleurs à une attitude de restauration des droits sociaux des travailleurs. G. Ricci, «I diritti sociali fra politiche di austerità e ripresa economica », DLM, 2016-1, p. 65. 


\section{A - PAUVRETÉ, TRAVAIL PRÉCAIRE ET TRAVAILLEUR À FAIBLE REVENU}

\section{1 - LA PAUVRETÉ APRÈS LA CRISE FINANCIÈRE DE 2008}

La crise financière de 2008 a frappé les pays de manière inégale. Parmi les pays européens, le taux de pauvreté de la population connaissant de graves difficultés matérielles a considérablement augmenté dans certains pays (Espagne, Grèce, Irlande et Italie), tandis que d'autres pays - en particulier la plupart des anciens pays membres de I'UE 15 - n'ont pas connu de changement important ${ }^{71}$. Ce contexte peut expliquer le fort intérêt des auteurs italiens de l'IALLJ pour la question de la pauvreté ${ }^{72}$, ainsi que pour les réformes de la politique sociale qui ont été mises en œuvre, au moins en partie en réponse à l'augmentation du taux de pauvreté. Les recherches menées en Italie sur la pauvreté ont accordé une attention particulière aux perspectives familiales et régionales. De nombreux pays (dont l'Italie) ont abordé les questions relatives aux travailleurs pauvres et précaire., Mais en Italie, on s'est intéressé particulièrement au taux de pauvreté élevé des enfants, en particulier dans le sud du pays ${ }^{73}$. Cette caractéristique pourrait expliquer en partie le vif intérêt des auteurs italiens pour l'amélioration du système national de protection sociale (voir ci-dessous) qui peut couvrir directement et de manière exhaustive tous les citoyens vivant dans la pauvreté dans l'ensemble du pays ${ }^{74}$.

\section{2 - LES DIFFÉRENTS TYPES DE TRAVAILLEURS PRÉCAIRES}

Une façon pour les auteurs de traiter la question de la pauvreté et du faible revenu était de la considérer sous l'angle des différents types de travail précaire offrant un niveau de salaire insuffisant. La question de l'emploi précaire a été traitée à plusieurs reprises par les auteurs de l'IALLJ après la récession et dans le contexte des politiques de flexibilité (flexisécurité $)^{75}$. En Europe, sauf dans les pays nordiques, la politique de flexibilité a entraîné une augmentation du nombre de travailleurs pauvres. Un numéro spécial a été consacré aux conséquences de cette politique en $2016^{76}$ dont une des contributions analyse la croissance de l'emploi précaire en Europe (15 anciens Etats membres de l'UE) comme conséquence de cette politique ${ }^{77}$. En se concentrant sur deux dimensions de

71 ECFIN Economic Brief, 2014, n³1, p. 3.

72 Ch. Saraceno, "Povertà ed esclusione sociale oggi in Italia: cause e possibili rimedi », RGL, 2016, 4, p. 717, G. Bronzini, P. Campanella, " Il lavoro tra bisogno e libertà: nuove povertà prospettive di inclusione sociale reddito e attivazione: Introduzione ", RGL, 2016-4, p. 687. Voir également M. Jessoula, "Italy's Winding Road Towards a National Minimum Income Scheme ", ESPN Flash Report, 2016/60.

73 C. Saraceno, op. cit.

74 Un autre élément de contexte pour expliquer cet intérêt pour les systèmes de protection sociale pourrait être l'absence de mesure nationale et universelle pour combattre la pauvreté en Italie. Sur ce point, voir S. La Forgia, « Le politiche regionali di contrasto alla povertà e di inserimento sociale e lavorativo ", RGL, $\mathrm{n}^{\circ} 3$, p. 574.

75 Voir S. Borelli, H. M. Hohnerlein, "Retrospective Overview of 2013 - Comparative labour law literature ", RDCTSS, 2014/3, p. 218, M. Aimo, D. Izzi, "Retrospective Overview of 2012 Comparative labour law literature », RDCTSS, 2013/3, p. 185.

76 ILR, 2016, vol. 155, n 4 (Numéro spécial : "The Age of Flexibility »).

77 T. Gutiérrez-Barbarrusa, «The growth of precarious employment in Europe: Concepts, indicators and the effects of the global economic crisis », ILR, 2016, vol. 155, n 4, p. 477. 
l'emploi précaire (insécurité et pauvreté -bas salaires ${ }^{78}$ ), l'auteur montre que la proportion de travailleurs à bas salaire est élevée dans les pays de l'Europe du Sud (Espagne, Grèce, Italie et Portugal), ainsi que dans les pays avec des marchés du travail plus déréglementés (Allemagne, Irlande, Pays-Bas et Royaume-Uni), tandis que les pays du Sud de l'Europe se caractérisent également par des niveaux élevés d'insécurité (mesurée en fonction de l'emploi atypique) $)^{79}$.

Plusieurs questions liées aux travailleurs précaires ont particulièrement intéressé les auteurs de l'IALLJ en 2016 : les questions liées à l'éducation, à la vie personnelle, au travail indépendant et aux activités liées à l'allocation de minima sociaux ${ }^{80}$.

Premièrement, certains auteurs se sont intéressés aux activités se situant entre l'éducation et le travail, situations de plus en plus fréquentes sur le marché du travail actuel, en particulier chez les jeunes travailleurs, dans les pays développés. On les appelle souvent des " apprentis ${ }^{81}$ ou des "stagiaires " ${ }^{82}$. Même si ces activités peuvent être extrêmement utiles pour les travailleurs comme pour les employeurs, elles peuvent engendrer des situations d'exploitation lorsque les employeurs remplacent à moindre coût de cette manière des travailleurs. Ils peuvent également être à l'origine de conditions injustifiables pour les stagiaires lorsque (comme c'est le cas dans de nombreux pays), il n'existe pas de réglementation suffisante pour la protection de leurs droits collectifs et individuels. Le Conseil de I'UE a adopté une Recommandation sur un cadre de qualité pour les stages en $2014^{83}$. Les auteurs de l'IALLJ présentant les situations de la législation et de la réglementation de I'UE ainsi que celles de certains pays de I'UE et hors UE (Etats-Unis, Australie, Royaume-Uni, Finlande, France et Estonie), partagent le point de vue selon lequel les lois et règlementations, à l'échelle européenne et nationale, n'offrent pas de protections suffisantes pour les stagiaires. Les efforts législatifs de la France pour protéger les stagiaires par la « loi Cherpion ${ }^{84}$ sont positivement appréciés par les auteurs, même si le manque de suivi et d'application est souligné ${ }^{85}$.

78 L'auteur adopte la définition Eurostar des bas salaires (salaires équivalant aux deux tiers ou moins des salaires horaires médians nationaux).

79 L'auteur compare les situations de 1995-2007 avec celles de 2008-2015. Outre l'influence de la politique de déréglementation, l'auteur souligne l'impact de l'expansion de l'UE en 2004 et 2007, en particulier pour l'aggravation de la pauvreté en Allemagne et au Royaume-Uni.

80 Parmi les autres types de travail précaire, le travail non déclaré a également attiré l'attention de certains auteurs. Voir M. Giaconi, « Le politiche europee di contrasto al lavoro sommerso. Tra (molto) soft law e (poco) hard law », LD, 3-2016, p. 439, S. Varva, "Undeclared work e Unione europea, ultimo atto: una "piattaforma europea" per contrastare il lavoro sommerso », LD, 3-2016, p. 461.

81 A. Rosin, "Precariousness of Trainees Working in the Framework of a Traineeship Agreement », IJCLLIR, 2016, vol. 32, n², p. 131.

82 R. Owens, A. Stewart, «Regulating for decent work experience: Meeting the challenge of the rise of the intern », ILR, 2016, vol. 155, $n^{\circ} 4$, p. 679.

83 L'OIT définit le stage comme " une période de travail limitée, rémunérée ou non, qui comprend une composante d'apprentissage et de formation, destinée à acquérir une expérience pratique et professionnelle en vue d'améliorer l'employabilité et de faciliter la transition vers un emploi régulier $"$.

84 Loi n²011-893 du 28 juillet 2011 pour le développement de l'alternance et la sécurisation des parcours professionnels.

85 R. A. Stewart, op. cit., p. 689. 
Deuxièmement, un numéro spécial a été publié sur les travailleurs qui doivent être " disponibles en sus de leur temps de travail ${ }^{86}$. Pour certains travailleurs (travailleurs occasionnels et de garde, mais aussi travailleurs à temps plein ou à temps partiel auxquels on demande régulièrement de travailler plus que les heures de travail convenues), la durée du travail et les horaires sont devenus de plus en plus imprévisibles, car ils doivent s'adapter aux besoins de production variables des employeurs ${ }^{87}$. Une étude sur les réseaux de services de soutien à domicile au Québec, au Canada, montre que ce nouveau type de travail est souvent pratiqué par des femmes et aboutit à un travail précaire et très mal rémunéré ${ }^{88}$.

Troisièmement, comme nous l'avons déjà vu ${ }^{89}$, le «travail de la foule (crowd work) » a également suscité beaucoup d'intérêt chez les auteurs de l'IALLJ en 2016. Ce type de travail peut également être considéré comme un nouveau type de travail précaire ainsi qu'il est présenté dans un article sur une enquête de I'OIT $^{90}$. Cet article propose d'assurer un salaire minimum pour ce type de travail, en maintenant le système de rémunération à la pièce. Le problème recoupe la question de la fixation des limites du temps de travail parce que ces travailleurs passent souvent beaucoup de temps en ligne, essayant de saisir un meilleur travail quand une offre est diffusée ${ }^{91}$.

Les auteurs ont également abordé une autre question rarement traitée concernant le statut précaire des bénéficiaires des programmes d'emploi dans le cadre de l'allocation de minima sociaux au Royaume-Uni et aux Pays-Bas, deux pays qui ont joué un rôle important dans les «programmes d'activation » en Europe et dans les autres pays développés ${ }^{92}$. Vu le développement continu des politiques d'activation dans les régimes de protection sociale dans de nombreux pays, cet article pourrait inspirer des recherches comparatives.

Comme le travail précaire suppose souvent un faible revenu, la question de la précarité recoupe largement celle de la pauvreté bien que celle-ci ait été moins souvent explorée par les auteurs de I'IALLJ93. Cependant, comme nous le verrons, de nombreux auteurs se sont intéressés à des questions diverses concernant les salaires, souvent en relation avec la question de la pauvreté.

86 IJCLLIR, 2016, vol. 32, n 3. Pour une approche théorique dans ce numéro, voir. É. Genin, «Proposal for a theoretical framework for the analysis of time porosity », p. 280.

87 G. Valée, «Employees' obligation to be available to Employers: A (New) Pathway to Precariousness or Source of Flexibility?», IJCLLIR, vol. 32, n³, p. 275.

88 L. Boivin, " "Just-in-Time" Labour: The case of networks providing home support services in Quebec ", IJCLLIR, 2016, n³, p. 301.

89 See supra, text \& notes at nn. 42-69.

90 J. Berg, «Income Security in the On-Demand Economy: Findings and Policy Lessons from a Survey of Crowdworkers», CLLPJ, 2016, vol. 37, p. 543.

91 J. Berg, op. cit., p. 561.

92 A. Paz-Fuchs \& Anja Eleveld, «Workfare revisited», ILJ, 2016, vol. 45, p. 29.

93 See F.J. Calvo Gallego, "Trabajadores pobres y pobreza de los ocupados: Una primera aproximación », $T L, n^{\circ} 134 / 2016$, p. 63. La difficulté de définir les «travailleurs pauvres » soulignée dans cet article pourrait être l'une des raisons de la réticence (relative) des chercheurs en droit à aborder ce sujet. 


\section{B - SALAIRES, SALAIRE MINIMUM ET SALAIRE DE SUBSISTANCE}

Des questions diverses concernant les salaires ont intéressé beaucoup d'auteurs de I'IALLJ en 2016, notamment la question du salaire minimum. L'introduction d'un salaire minimum peut parfois être considérée comme une des solutions au problème de la pauvreté, car il peut augmenter le revenu des travailleurs précaires vivant souvent dans la pauvreté. II est également considéré comme une politique économique qui soutient et encourage la consommation nationale et contribue ainsi au développement économique. II est donc logique qu'après la crise économique et financière mondiale de 2008, la question des salaires ait été considérée comme urgente par les chercheurs dans de nombreux pays de différents continents, mais aussi par des organisations internationales ${ }^{94}$. L'introduction d'un salaire minimum légal en Allemagne ${ }^{95}$ et les débats sur la possibilité d'un salaire minimum européen ${ }^{96}$ ont renforcé cet intérêt, notamment en Europe. Dans le contexte de l'Union européenne, le salaire minimum pourrait également être considéré comme un outil permettant de créer un environnement concurrentiel équitable du point de vue des coûts de la main-d'œuvre. Enfin, une perspective relativement nouvelle a également été présentée concernant le salaire minimum : le concept de "salaire de subsistance (living wage)». Les auteurs qui plaident pour un salaire de subsistance se concentrent sur les droits de l'homme et la justice sociale pour déterminer le niveau minimum de salaire qui devrait être garanti, considérant le « salaire minimum » tel qu'il est généralement conçu aujourd'hui comme insuffisant de ce point de vue.

\section{1 - LE SALAIRE MINIMUM}

Autour du salaire minimum se posent de nombreuses questions: son niveau, le processus de détermination, les effets sur le marché du travail, l'efficacité, etc. Concernant le processus de décision, du point de vue du droit du travail comparé, il est essentiel de prendre en considération le rôle des partenaires sociaux (y compris les partenaires sociaux au niveau européen en ce qui concerne les réglementations européennes). Un auteur autrichien discute ce point en comparant les lois allemandes et autrichiennes et les relations des partenaires sociaux, affirmant que dans les pays où la couverture de la négociation collective est élevée, le salaire minimum collectivement négocié peut garantir des salaires minima décents pour les salariés et, en même temps, garantir un minimum de

94 COM (2012)173, p. 9, S. C. Carr, J. Parker, J. Arrowsmith, P. A. Watters, «The living wage: Theoretical integration and an applied research agenda », ILR, 2016, vol. 155, n 1, p. 1 (voir en particulier l'introduction de l'article concernant les débats récents sur les salaires).

95 Th. Lakies, «Ein Jahr gesetzlicher Mindestlohn - gelöste und ungelöste Rechtsprobleme », AUR, 2016 - 1, p. 14. H. Pütz, "Zur Vergütungspflicht von Überstunden durch das Mindestlohngesetz », AUR, 2016 - 5, p. 186. P. Rémy, "La loi allemande sur le salaire minimum : le SMIC en Allemagne?», RDT, 2014, p. 774.

96 Voir par exemple, Schulten, Thorsten, T. Muller, L. Eldring. «Pour une politique de salaire minimum européen : perspectives et obstacles », La Revue de I'Ires, 2016, vol. 89, n² 2, p. 89. 
liberté contractuelle pour les parties à la relation de travail97. Les effets de répartition et d'égalisation du salaire minimum intéressent les auteurs latino-américains. L'article sur les effets en termes de distribution de l'augmentation de la valeur réelle du salaire minimum en Argentine, au Brésil, au Chili et en Uruguay montre que les effets distributifs ont été confirmés dans trois de ces pays (tous sauf le Chili) ${ }^{98}$.

Différents facteurs influent sur l'efficacité du salaire minimum. Un auteur souligne, en examinant les situations dans les pays d'Amérique latine (Chili, Costa Rica, Pérou et Uruguay) que le salaire minimum doit s'accompagner d'un système d'inspection du travail efficace, en particulier lorsque le salaire minimum est très bas ou très élevé par rapport aux salaires moyens du pays ${ }^{99}$. Un auteur autrichien a également souligné que le salaire minimum sectoriel convenu collectivement est plus facilement appliqué par les employeurs que le minimum universel prévu par la loi ${ }^{100}$.

\section{2 - UNE NOUVELLE ORIENTATION POUR LE SALAIRE MINIMUM : LE SALAIRE DE SUBSISTANCE}

Une des questions majeures sur le salaire minimum est, bien sûr, de savoir quel devrait être le niveau : quel niveau est nécessaire et suffisant? Cette question ne peut pas être isolée de l'objectif recherché avec le salaire minimum. Même si, comme nous l'avons mentionné plus haut, le salaire minimum peut avoir plusieurs dimensions - droits des travailleurs, politique économique, concurrence loyale entre employeurs, etc., les auteurs de I'IALLJ ont presque tous traité ce sujet du premier point de vue, à savoir comme droit fondamental du travailleur, et, par extension, comme la traduction d'une certaine justice sociale. Dans un article inspiré de l'idée d'un salaire minimum européen et du système de salaire minimum allemand, un auteur affirme que le salaire devrait garantir une "rémunération juste et suffisante du travail réalisé $»^{101}$.

De ce point de vue, une nouvelle vision du salaire minimum - appelé (ou non) «salaire de subsistance (living wage) ${ }^{102}$ - qui devrait être fixé au niveau garantissant la dignité humaine et pas seulement la seule survie matérielle des travailleurs - a été proposé par certains auteurs. Cette tendance est fortement influencée par «l'approche en termes de capabilities» proposée par Amartya Sen et Martha Nussbaum ${ }^{103}$, une théorie qui intéresse beaucoup de spécialistes du droit du travail, étant considérée comme " pierre angulaire pour repenser la législation du travail ${ }^{104}$.

97 E. Brameshuber, " "Basic Income" from Wages: Freedom of Contract versus Statutory Minimum Standards », ELLJ, 2016, vol. 7, p. 206. L'auteur estime aussi que dans cette situation le niveau général de rémunération est relativement élevé. p. 227.

98 R. Maurizio, G. Vázquez, "Distribution effects of the minimum wage in four Latin American countries: Argentina, Brazil, Chile and Uruguay », ILR, 2016, vol. 155, p. 97.

99 A. Marinakis, "Non-compliance with minimum wage laws in Latin America: The importance of institutional factors », ILR, 2016, vol. 155, p. 133.

100 E. Brameshuber, op. cit., pp. 226 and 227.

101 E. Brameshuber, op. cit.

102 S. C. Carr, J. Parker, J.Arrowsmith, P. A. Watters, op. cit.

103 Voir par exemple, M. Nussbaum, Creating Capabilities: The Human Development Approach, Cambridge, MA, Harvard University Press, 2011.

104 R. Del Punta, "Labour Law and the Capability Approach », IJCLLIR, 2016, p. 383. 
Dans les articles débattant de le niveau minimum de salaires, différents termes sont utilisés par différents acteurs et auteurs, qui ne sont pas toujours strictement définis et qui peuvent être utilisés dans différents sens - équitable » ${ }^{105}$, " juste revenu », " rémunération suffisante du travail », « revenu de base », "salaire minimum (revenu) », "salaire décent » ou " nivau de revenu (...) pour vivre dans la dignité », etc. ${ }^{106}$. De plus, la notion de "revenu » peut recouvrir soit les seuls salaires, soit les salaires et la sécurité sociale ${ }^{107}$. Ceci nous incite à déterminer précisément le sens des termes utilisés à propos de salaire minimum. Le caractère complexe et multifonctionnel du salaire minimum justifie également une attitude prudente dans les débats y relatifs.

\section{3 - AUTRES QUESTIONS SUR LES SALAIRES}

L'intérêt grandissant accordé au salaire minimum pourrait également accroître l'intérêt pour différentes questions sur les salaires en général ${ }^{108}$. Et en particulier, certaines questions mériteront d'être examinées dans une perspective comparative, comme celle sur l'efficacité des différentes normes sur les salaires en général ${ }^{109}$ ou le rôle de l'inspection du travail dans la définition des salaires. Sur une question plutôt classique mais toujours controversée, un auteur a examiné dans une étude comparative le traitement juridique des " pourboires » en France, en Allemagne et aux Etats-Unis ${ }^{110}$.

\section{C - REVENU DE BASE ET AIDE SOCIALE}

Même si le salaire minimum peut résoudre une partie du problème de la pauvreté, ce n'est évidemment pas une solution magique pour toutes les situations de pauvreté. Premièrement, le salaire minimum est souvent fixé à un niveau insuffisant pour garantir les conditions de vie au-delà du seuil de pauvreté ${ }^{111}$. De plus, si le salaire minimum est fixé sur une base horaire, ce n'est pas une solution au problème de l'insuffisance du temps de travail (typiquement les travailleurs à temps partiel aux revenus modestes). Inutile de dire que le salaire minimum ne peut couvrir que la population active et que le problème de la pauvreté de ceux qui ne travaillent pas ou qui ne sont pas en âge de travailler ne pourra jamais être totalement résolu par un programme établissant un salaire minimum ou un salaire de subsistance.

105 E. Brameshuber, op. cit., p. 213.

106 Selon l'article, ce concept est lié au concept plus large de « travail décent » inclus et défini dans l'agenda de I'OIT. (voir S. C. Carr et al., op. cit.). À propos du travail décent, voir aussi Fr. D'Ovidio, "Il lavoro che verrà », RGL, 2016-4, p. 698.

107 E. Brameshuber, op. cit., p. 210.

108 La Revue internationale du Travail a publié un numéro spécial sur les salaires, 2016, vol. 155-1. Entre autres choses, le numéro comprend une analyse de l'inégalité des salaires en Chine. J. Whalley, Ch. Xing, "Ownership restructuring and wage inequality in urban China », ILR, 2016, 155-1, p. 57.

109 Voir les articles dans le vol. 37, n², CLLPJ, et également R. Bignami, M. C. Serrano Barbosa, « La Inspección de Trabajo y el fraude salarial en Brasil: justicia al nivel de la calle, desarrollo y paz social », DRL, 2016-7, p. 686.

110 M. W. Finkin, «The Kindness of Strangers: The Tip and the Minimum Wage in France, Germany, and the United States », IJCLLIR, 2016, vol. 32, n 1, p. 3.

111 E. Brameshuber, op. cit., p. 210. 
En conséquence, différents types de régimes de protection sociale -assistance sociale et prestations de chômage- ont aussi intéressé les auteurs de I'IALLJ comme solutions possibles au problème de la pauvreté ${ }^{112}$. Dans le mouvement général et continu des politiques d'aide à l'emploi dans les régimes de protection sociale d'un grand nombre de pays, une orientation opposée a été examinée par certains auteurs : le revenu de base universe $\left.\right|^{113}$. Les auteurs, en présentant différents types de revenu de base universel, ont souligné les limites du concept : son uniformité et le faible niveau d'allocation par rapport aux prestations de sécurité sociale existantes, qui varient selon la nécessité réelle des individus. Selon les auteurs, le concept de revenu de base universel est présenté comme pouvant englober divers types de systèmes de garantie de revenu, y compris de nombreuses prestations sociales existantes ${ }^{114}$.

112 Voir par exemple, M. Arai, "Gesetz über die Soziale Wohlfahrt und Rechtsschutz für Nutzer von sozialen Wohlfahrtsdiensten », ZIAS, 2016, Heft 1, 30. Jahrgang, 2016, p. 3 ; U. Kötter, « Die Sozialhilfe im Recht der Europäischen Union - immer noch eine Randexistenz oder auf dem Weg zu einem "Europäischen Sozialhilferecht"? », ZIAS, 2016, Heft 1, 30. Jahrgang, 2016 p. 98 ; S. Spattini, "Sostegno al reddito dei disoccupati nella legislazione nazionale ", RGL, 2016-3, p. 550-561; N. Paci, « Disoccupazione e strumenti di sostegno del reddito nel Jobs Act? », RGL, 2016-4, p. 781 ; G. Viña, Jordi, «La protección por desempleo en España », DRL, n 5, p. 425 ; B. Suárez Corujo, "El colapso de la protección por desempleo en tiempos de crisis », DRL, 2016, $n^{\circ} 7$, p. 655 ; A. Occhino, « Il sostegno al reddito dei lavoratori in costanza di rapporto tra intervento pubblico e bilateralità », DLM, 2016, vol. 3, p. 499.

113 Un auteur italien affirme que le revenu de base devrait être considéré comme un outil constitutionnellement nécessaire à l'interprétation de la Constitution italienne. Ch. Tripodina, "Povertà e dignità nella Costituzione italiana: il reddito di cittadinanza come strumento costituzionalmente necessario », RGL, 2016-4, p. 732.

114 Gr. Strban, R. Mihalič, Prednosti in pomanjkljivosti različnih oblik univerzalnega temeljnega dohodka, E\&E, vol. 16, n' $1 / 2016$, p. 9. 2 Dans cet article, la forme originale et fondamentale d'un revenu de base universel est définie comme un revenu non sélectif, durable, individuel et totalement inconditionnel, indépendant de la situation sociale et économique du bénéficiaire et de l'origine de la situation sociale. 


\section{LitTÉRATURE DE DROIt SOCIAL COMparé}

\section{List of journal abbreviations}

Arbeit und Recht (Germany) $=$ AuR

Australian Journal of Labour Law (Australia) $=$ AJLL

Bulletin of Comparative Labour Relations (Belgium) $=$ BCLR

Canadian Labour \& Employment Law Journal (Canada) $=$ CLELJ

Comparative Labor Law \& Policy Journal (United States) = CLLPJ

Derecho de las Relaciones Laborales $=$ DRL

Diritti Lavori Mercati (Italy) = DLM

Employees \& Employers: Labour Law \& Social Security Review (Slovenia) $=\mathrm{E} \& \mathrm{E}$

Europäische Zeitschrift für Arbeitsrecht (Germany) $=$ EuZA

European Labour Law Journal (Belgium) = ELLJ

Giornale di Diritto del Lavoro e delle Relazioni Industriali (Italy) = DLRI

Industrial Law Journal (UK) = ILJ

International Journal of Comparative Labour Law \& Industrial Relations

(The Netherlands) $=$ IJCLLIR

International Labour Review (ILO) = ILR

Revue Internationale de Travail (France) $=$ RIT

Japan Labor Review (Japan) = JLR

Lavoro e Diritto (Italy) = LD

Pécsi Munkajogi Közlemények (Pecs Labour Law Journal) (Hungary) = PMJK

Relaciones Laborales (Spain) $=\mathrm{RL}$

Revista de Derecho Social (Spain) = RDS

Revue de Droit Comparé du Travail et de la Sécurité Sociale (France) = RDCTSS

Revue de Droit du Travail (France) $=$ RDT

Revista Giuridica del Lavoro e della Previdenza Sociale $=$ RGL

Temas Laborales (Spain) $=T L$

Zeitschrift für ausländisches und internationales Abreits und Socialrecht (Germany) = ZIAS 